\title{
Celiac axis stenosis due to median arcuate ligament compression in a patient who underwent pancreatoduodenectomy; intraoperative assessment of hepatic arterial flow using Doppler ultrasonography: a case report
}

\author{
Masateru Yamamoto ${ }^{1}$, Toshiyuki Itamoto ${ }^{1,2^{*}}$, Akihiko Oshita ${ }^{1,2}$ and Yasuhiro Matsugu ${ }^{1}$
}

\begin{abstract}
Background: Celiac axis stenosis due to compression by the median arcuate ligament has been reported in patients undergoing pancreaticoduodenectomy; it leads to the development of major collateral pathways that feed the hepatic artery. Dividing these important collaterals during pancreaticoduodenectomy can cause ischemic complications which may lead to a high mortality rate. To prevent these complications, it is necessary to assess intrahepatic arterial flow.

Case presentation: A 71-year-old Japanese man with anorexia was referred to us for the treatment of alcoholic chronic pancreatitis. Computed tomography revealed a pancreatic head tumor with a calculus, associated with the dilatation of the main pancreatic duct and intrahepatic bile duct. Three-dimensional imaging demonstrated focal narrowing in the proximal celiac axis due to median arcuate ligament compression and a prominent gastroduodenal artery that fed the common hepatic artery. The preoperative diagnosis was alcoholic chronic pancreatitis with common bile duct obstruction and celiac axis stenosis due to median arcuate ligament compression. Pancreaticoduodenectomy with median arcuate ligament release was scheduled. Before the division of the median arcuate ligament, the peak flow velocity and resistive index of his intrahepatic artery measured with Doppler ultrasonography decreased from $37.7 \mathrm{~cm} / \mathrm{second}$ and 0.510 , respectively, to $20.6 \mathrm{~cm} / \mathrm{second}$ and 0.508 respectively, when his gastroduodenal artery was clamped. However, these values returned to baseline levels after the division of the median arcuate ligament. These findings suggested that pancreaticoduodenectomy could be performed safely. Our patient was discharged on postoperative day 17 without significant complications.

Conclusion: The intraoperative quantitative evaluation of intrahepatic arterial blood flow using Doppler ultrasonography was useful in a patient who underwent pancreaticoduodenectomy, who had celiac axis stenosis due to compression by the median arcuate ligament.
\end{abstract}

Keywords: Celiac axis stenosis, Doppler ultrasonography, Median arcuate ligament, Pancreatoduodenectomy, Hepatic arterial flow, Resistive index

\footnotetext{
* Correspondence: t-itamoto@hph.pref.hiroshima.jp

'Department of Gastroenterological, Breast and Transplant Surgery,

Hiroshima Prefectural Hospital, Hiroshima, Japan

2Department of Gastroenterological and Transplant Surgery, Applied Life

Science, Institute of Biomedical and Health Science, Hiroshima University,

Hiroshima, Japan
} 


\section{Background}

Blood supply to the abdominal viscera mainly arises from the celiac artery and superior mesenteric artery (SMA). The pancreatic head region is the most important site of communication between these two arterial systems via the gastroduodenal artery (GDA) and the pancreaticoduodenal arcades, respectively. Pancreaticoduodenectomy (PD) involves the division of GDA and resection of the pancreaticoduodenal arcades, which depend on both GDA and SMA.

Celiac axis stenosis (CAS) caused by external compression or internal occlusion has been reported in 2 to $7.6 \%$ of patients undergoing PD [1-6]. Compression by the median arcuate ligament (MAL) is the most common cause of CAS [6]. In CAS, the division of GDA in PD can cause ischemic complications including anastomotic dehiscence, liver failure, and liver abscess. All complications lead to a high rate of mortality, especially if the collateral pathways from SMA are inadequate [6]. To prevent these serious complications, the GDA clamping test is mandatory before the division of GDA, ensuring that sufficient hepatic blood flow is preserved by ascertaining the satisfactory pulsation of the proper hepatic artery and/or by Doppler ultrasonography. Poor arterial perfusion in the liver during the clamping test necessitates MAL division with or without additional reconstruction of the celiac artery to restore blood flow to the liver. To the best of our knowledge, no reports have described quantitative criteria for hepatic blood flow restoration using Doppler ultrasonography.

Here, we report a case of PD performed safely with quantitative intrahepatic arterial flow evaluation using intraoperative Doppler ultrasonography.

\section{Case presentation}

A 71-year-old Japanese man with chronic pancreatitis and pancreatic diabetes, who complained of anorexia was referred to our hospital. Laboratory findings on admission included total bilirubin, direct bilirubin, albumin, and glycated hemoglobin (hemoglobin A1c) which showed values of $1.6 \mathrm{mg} / \mathrm{dL}, 0.9 \mathrm{mg} / \mathrm{dL}, 3.5 \mathrm{~g} / \mathrm{dL}$, and $10.3 \%$, respectively; Aspartate and alanine aminotransferase, alkaline phosphatase, and gamma-glutamyl transpeptidase showed concentrations of $63 \mathrm{U} / \mathrm{L}, 141 \mathrm{U} / \mathrm{L}$, $942 \mathrm{U} / \mathrm{L}$, and $1076 \mathrm{U} / \mathrm{L}$, respectively. Serum carbohydrate antigen 19-9 was elevated $(112 \mathrm{U} / \mathrm{mL})$. Computed tomography $(\mathrm{CT})$ showed a calculus within the swollen pancreatic head (Fig. 1a), which had caused dilatation of the common bile duct, intrahepatic bile duct, and main pancreatic duct (Fig. 1b). These findings were clarified by endoscopic retrograde cholangiopancreatography. Endoscopic ultrasonography (EUS) revealed a low echoic mass (40 $\mathrm{mm}$ in diameter) in the pancreatic head in which a strong echo with acoustic shadow was detected.
EUS-guided fine-needle aspiration cytology for the pancreatic head tumor showed no malignancy. Threedimensional volume-rendered imaging demonstrated a prominent GDA that fed the common hepatic artery of his SMA, indicating that celiac arterial flow might cease or reduce (Fig. 2a). A sagittal maximum-intensity projection $\mathrm{CT}$ angiogram and three-dimensional volume-rendered imaging demonstrated acute angulation and narrowing of the proximal celiac axis (CA) resulting in poststenotic dilatation, which created a "hooked" appearance (arrow) that was characteristic of MAL compression (Fig. 2b, c).

Based on these findings, the preoperative diagnosis was symptomatic mass-forming chronic pancreatitis and CAS due to MAL compression; thus, PD with or without MAL release was scheduled. The GDA clamping test was performed by measuring the intrahepatic arterial flow using Doppler ultrasonography before GDA division. Waveform did not change to tardus-parvus pattern after clamping the GDA. The peak systolic and mean velocities of the intrahepatic arterial flow decreased from $37.7 \mathrm{~cm} /$ second and $26.4 \mathrm{~cm} /$ second, respectively, before GDA clamping, to $20.6 \mathrm{~cm} /$ second and $15.0 \mathrm{~cm} /$ second, respectively, after GDA clamping. Similarly, the resistive index (RI) decreased from 0.510 to 0.508 (Fig. 3a, b). These changes were important and indicated the necessity for MAL release to minimize ischemic complications in our patient's liver. MAL release was performed as follows. The right diaphragmatic crus was progressively divided on the right side of his abdominal aorta at the level of SMA origin. Thereafter, the right side and the upper edge of the CA were progressively freed of all dense fibrous tissue. MAL release restored the peak systolic and mean velocities of intrahepatic blood flow during the GDA clamping to $34.4 \mathrm{~cm} /$ second and $23.8 \mathrm{~cm} /$ second, respectively. RI was also restored to 0.512 , enabling us to divide GDA safely and to perform a subsequent dissection of the pancreas head (Fig. 3c).

His postoperative course was uneventful, and he was discharged on postoperative day 17. Abdominal CT on postoperative day 30 showed that there was no stenosis at the CA (Fig. 4).

\section{Discussion and conclusions}

External compression at the origin of the CA may occur and is mainly due to an anomaly of MAL. MAL is a fibrous arch that unites the diaphragmatic crura on either side of the aortic hiatus. The ligament usually passes over the aorta and superior to the origin of the CA; however, it passes at the level of or below the origin of the CA in up to $33 \%$ of fresh autopsy specimens [7] and may lead to the symptomatic 


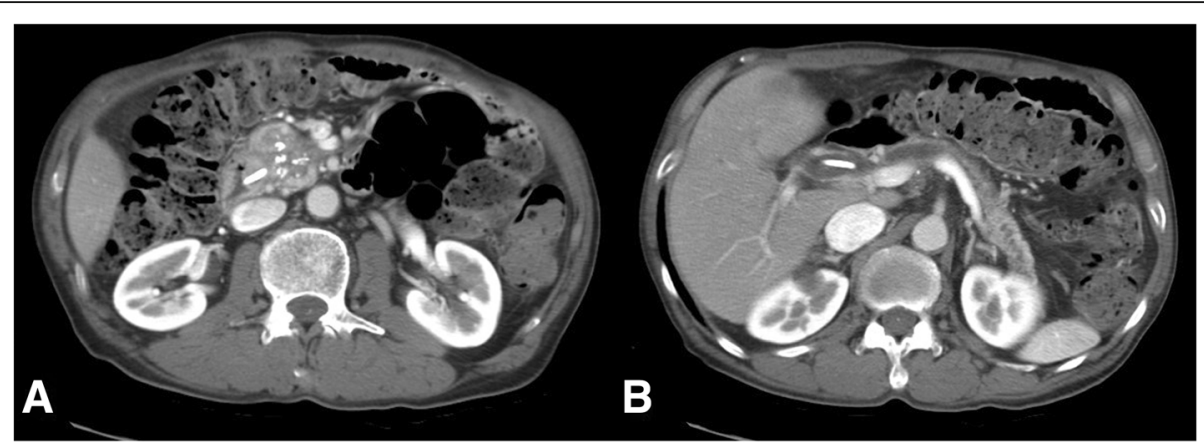

Fig. 1 Computed tomography findings. The calculus within the swollen pancreatic head (a) led to the dilatation of the common bile duct, intrahepatic bile duct, and main pancreatic duct (b)

compression of the CA, that is, MAL syndrome, which was first described in 1917 [8].

It has become possible to detect uncommon abnormalities using recent imaging techniques. Three-dimensional CT angiography makes it possible to diagnose CAS caused by various etiologies with $96 \%$ sensitivity and to determine the etiology of CAS with $92 \%$ accuracy [6]. CAS caused by MAL compression often exhibits, on imaging, more than $50 \%$ stenosis approximately $5 \mathrm{~mm}$ distal to the celiac artery bifurcation [6]. Hooked appearance is a characteristic finding in which the origin of CA is deformed into a hook shape [9]. CAS results in the development of major collateral pathways (pancreaticoduodenal arcades or the dorsal pancreatic artery) which arise from SMA, resulting in the feeding of the branches of the common hepatic artery through retrograde flow via GDA or the arc of Buhler [10-12]. Dividing these important collaterals to the upper abdominal organs during PD in a patient with CAS can cause postoperative life-threatening ischemic complications including hepatic and anastomotic ischemia [13, 14].

To prevent these serious complications, CAS caused by MAL compression can be treated by complete MAL division during PD. However, some investigators have argued that the division of GDA during PD in patients with CAS does not always result in ischemic complications of the upper abdominal organs [1-3], reporting that only 13 to $17 \%$ of patients with CAS required arterial reconstruction during PD, because abundant collateral anastomoses other than the pancreatic head arcade might develop between the CA tributaries and SMA tributaries [1,3]. Song et al. also reported that in patients with CAS, collateral pathways, such as from the dorsal pancreatic artery or replaced right hepatic artery, existed in approximately $80 \%$ of the patients [12]. However, Nara et al. reported that two of four patients with CAS, who did not undergo MAL division, developed liver abscesses after PD [15].

In cases of CAS, intraoperative trial clamping of GDA with the palpation of the hepatic artery or with the assessment of intrahepatic arterial flow by Doppler ultrasonography is of paramount importance before the division of GDA in order to prevent postoperative ischemic complications [3]. The weakening of hepatic arterial pulsation and the decrease of hepatic arterial flow by Doppler ultrasonography in the GDA clamping test indicate the necessity of MAL division during PD. Gaujoux et al. reported that CAS due to MAL was diagnosed in approximately $10 \%$ of the candidates for PD and

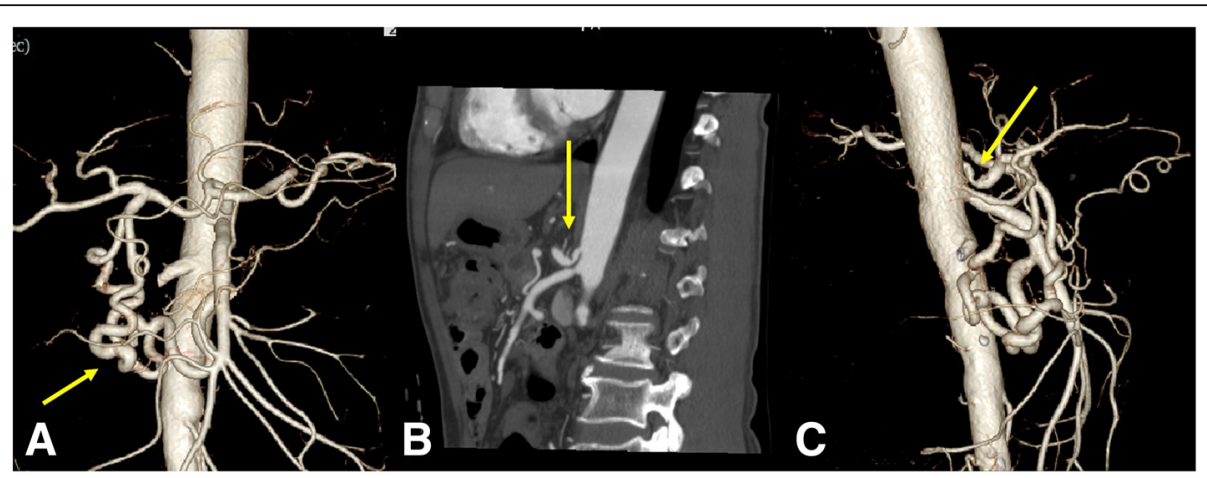

Fig. 2 Three-dimensional volume-rendered images. The prominent gastroduodenal artery that fed the common hepatic artery from superior mesenteric artery indicated that celiac arterial flow might cease or reduce (a). Acute angulation and narrowing of the proximal celiac axis resulted in poststenotic dilatation, which created a "hooked" appearance (arrow) (b, c) 


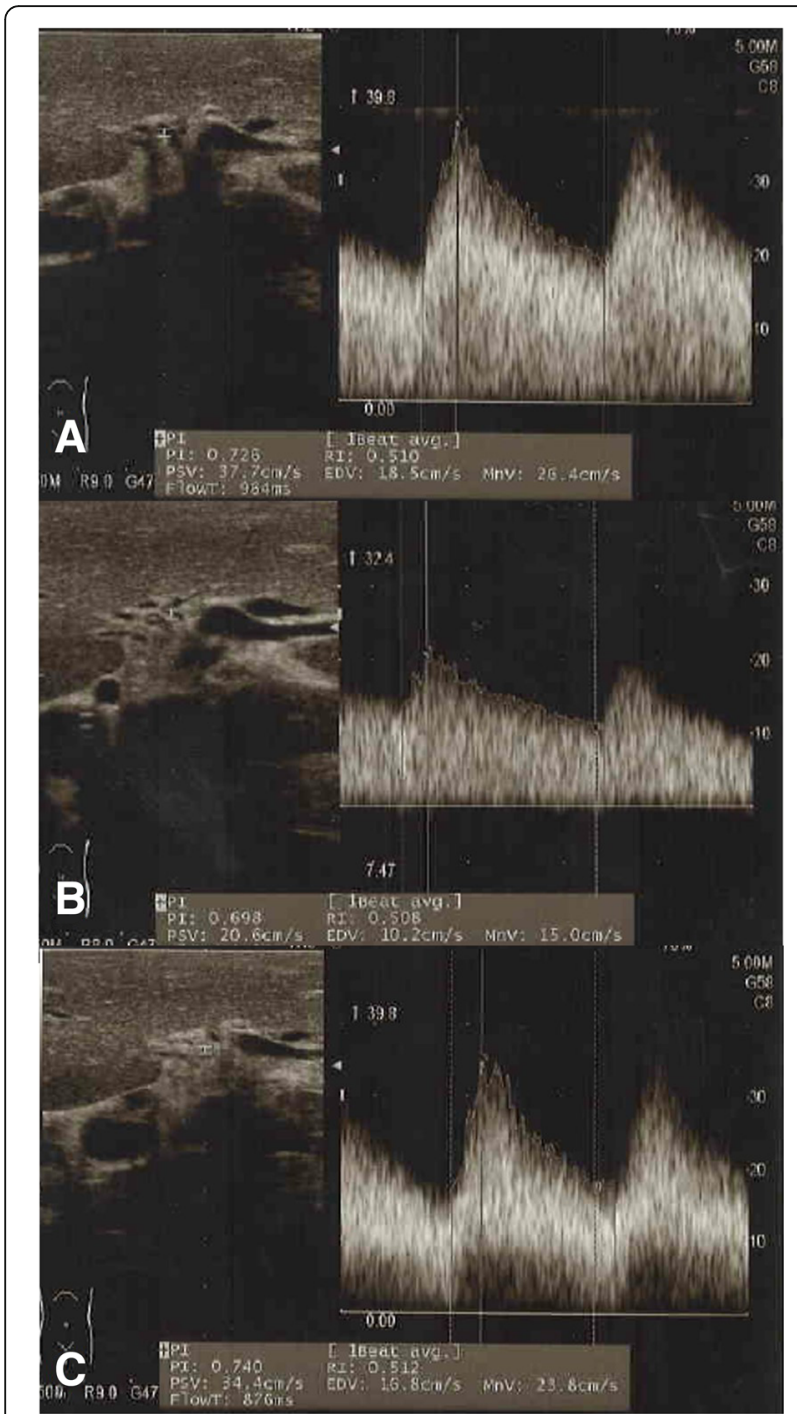

Fig. 3 Doppler ultrasonography for gastroduodenal artery clamping test. The peak systolic and mean velocities of intrahepatic arterial flow decreased from $37.7 \mathrm{~cm} /$ second and $26.4 \mathrm{~cm} / \mathrm{second}$, respectively, before gastroduodenal artery clamping to $20.6 \mathrm{~cm} / \mathrm{second}$ and $15.0 \mathrm{~cm}$ / second, respectively, after gastroduodenal artery clamping. Similarly, the resistive index decreased from 0.510 to 0.508 , respectively $(\mathbf{a}, \mathbf{b})$. After the median arcuate ligament was released, the peak systolic and mean velocities of intrahepatic blood flow were restored to $34.4 \mathrm{~cm} / \mathrm{second}$ and $23.8 \mathrm{~cm} / \mathrm{second}$, respectively, during gastroduodenal artery clamping. The resistive index also restored to 0.512 (c)

approximately half of these, who became candidates for MAL division, were found to have hemodynamically significant CAS during the GDA clamping test via Doppler assessment [6]. However, they did not show the threshold of a significant decrease in hepatic arterial blood flow. Nara et al. also described the need for intraoperative assessment using Doppler ultrasonography in patients with CAS during PD [15]. Decreased or absent hepatic arterial pulsation or signal using intraoperative Doppler ultrasonography would indicate the need for

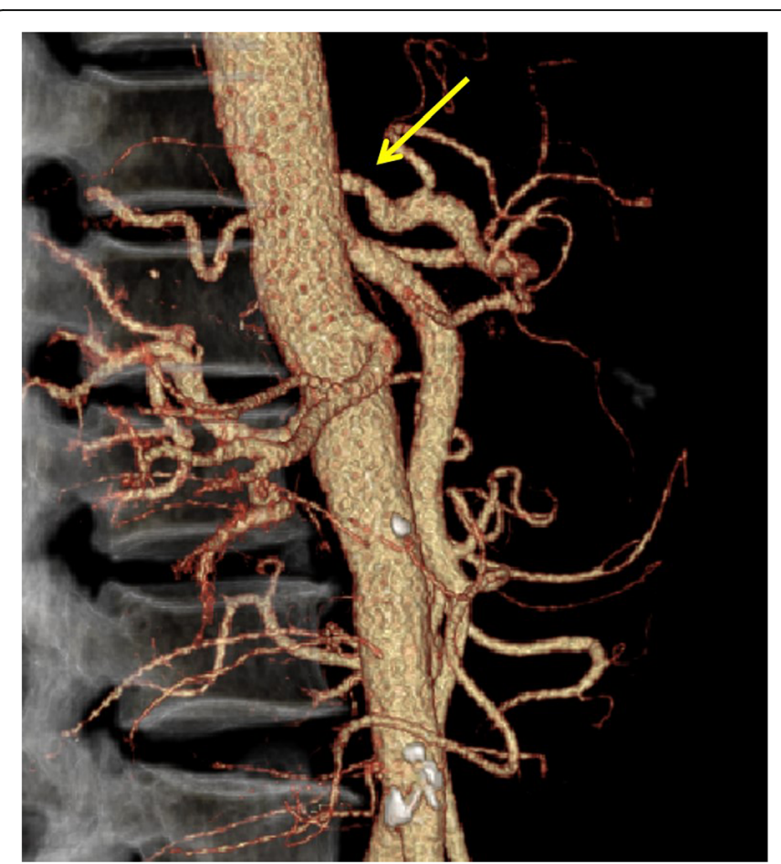

Fig. 4 Three-dimensional volume-rendered images on postoperative day 30. There was no stenosis of the celiac artery origin (arrow)

vascular reconstruction or MAL release. However, there is no consensus on the threshold beyond which MAL division or an additional intervention is required.

Doppler ultrasonography can evaluate blood flow not only qualitatively but also quantitatively in the field of liver transplantation [16, 17]. The normal hepatic artery Doppler waveform shows a rapid systolic upstroke following continuous diastolic flow. The acceleration time and RI can serve as indicators of hepatic arterial blood flow assessment. The acceleration time, which represents the time from the end of diastole to the first systolic peak, should be less than $80 \mathrm{~ms}$. The RI, which represents (peak systolic velocity - end diastolic velocity)/ peak systolic velocity, should be between 0.5 and 0.7 [16]. Tardus-parvus pattern of waveform with an acceleration time greater than $80 \mathrm{~ms}$ and RI less than 0.5 represent insufficient arterial flow due to hepatic artery stenosis in the setting of liver transplantation [17].

In the present case when GDA was clamped, the peak and mean velocities and RI decreased reproducibly compared with their baseline levels which were measured before clamping, although RI was maintained at more than 0.50 and the tardus-parvus pattern of waveform was not found. These decreased values almost returned to the baseline levels during GDA clamping just after MAL division.

To establish definitive criteria and to clarify the threshold for MAL division or for additional hepatic arterial reconstruction during PD for patients with CAS, a large number of patients should be studied based on the 
precise evaluation of the velocity of hepatic arterial inflow using Doppler ultrasonography. However, MAL division might be indicated when a reproducible decrease in hepatic arterial flow is noted during GDA clamping. Moreover, restoration of flow values to baseline levels by MAL division might imply the unnecessariness of additional hepatic arterial reconstruction.

\section{Abbreviations}

CA: Celiac axis; CAS: Celiac axis stenosis; CT: Computed tomography; EUS: Endoscopic ultrasonography; GDA: Gastroduodenal artery; MAL: Median arcuate ligament; PD: Pancreaticoduodenectomy; RI: Resistive index; SMA: Superior mesenteric artery

\section{Acknowledgements}

This study was not funded by any grants. We would like to thank Editage (www.editage.jp) for English language editing.

\section{Funding}

This study was not funded by any grants.

\section{Availability of data and materials}

Not applicable.

\section{Authors' contributions}

$\mathrm{MY}, \mathrm{TI}, \mathrm{AO}$ and YM drafted the manuscript. TI has given the final approval of the version to be published. All authors read and approved the final manuscript.

\section{Ethics approval and consent to participate}

The procedure was approved by the Ethics Committee at the Prefectural Hiroshima Hospital.

\section{Consent for publication}

Written informed consent was obtained from the patient for publication of this case report and any accompanying images. A copy of the written consent is available for review by the Editor-in Chief of this journal.

\section{Competing interests}

The authors declare that they have no competing interests.

\section{Publisher's Note}

Springer Nature remains neutral with regard to jurisdictional claims in published maps and institutional affiliations.

Received: 4 October 2017 Accepted: 12 February 2018

Published online: 11 April 2018

References

1. Trede M. The surgical treatment of pancreatic carcinoma. Surgery. 1985; 97(1):28-35.

2. Pfeiffenberger J, Adam U, Drognitz O, Kroger JC, Makowiec F, Schareck W, et al. Celiac axis stenosis in pancreatic head resection for chronic pancreatitis. Langenbeck's Arch Surg. 2002;387(5-6):210-5.

3. Berney $T$, Pretre $R$, Chassot G, Morel $P$. The role of revascularization in celiac occlusion and pancreatoduodenectomy. Am J Surg. 1998;176(4):352-6.

4. Portolani N, Tiberio GA, Coniglio A, Baiocchi G, Vettoretto N, Giulini SM. Emergency celiac revascularization for supramesocolic ischemia during pancreaticoduodenectomy: report of a case. Surg Today. 2004;34(7):616-8.

5. Farma JM, Hoffman JP. Nonneoplastic celiac axis occlusion in patients undergoing pancreaticoduodenectomy. Am J Surg. 2007;193(3):341-4 discussion 4

6. Gaujoux S, Sauvanet A, Vullierme MP, Cortes A, Dokmak S, Sibert A, et al. Ischemic complications after pancreaticoduodenectomy: incidence, prevention, and management. Ann Surg. 2009;249(1):111-7.

7. Lindner HH, Kemprud E. A clinicoanatomical study of the arcuate ligament of the diaphragm. Arch Surg. 1971;103(5):600-5.

8. Lipshutz B. A COMPOSITE STUDY OF THE COELIAC AXIS ARTERY. Ann Surg 1917;65(2):159-69.
9. Horton KM, Talamini MA, Fishman EK. Median arcuate ligament syndrome: evaluation with CT angiography. Radiographics. 2005;25(5):1177-82.

10. Kornblith PL, Boley SJ, Whitehouse BS. Anatomy of the splanchnic circulation. Surg Clin North Am. 1992;72(1):1-30.

11. Sakorafas GH, Sarr MG, Peros G. Celiac artery stenosis: an underappreciated and unpleasant surprise in patients undergoing pancreaticoduodenectomy. J Am Coll Surg. 2008;206(2):349-56.

12. Song SY, Chung JW, Kwon JW, Joh JH, Shin SJ, Kim HB, et al. Collateral pathways in patients with celiac axis stenosis: angiographic-spiral CT correlation. Radiographics. 2002;22(4):881-93.

13. Bull DA, Hunter GC, Crabtree TG, Bernhard VM, Putnam CW. Hepatic ischemia, caused by celiac axis compression, complicating pancreaticoduodenectomy. Ann Surg. 1993;217(3):244-7.

14. Thompson NW, Eckhauser FE, Talpos G, Cho KJ. Pancreaticoduodenectomy and celiac occlusive disease. Ann Surg. 1981;193(4):399-406.

15. Nara S, Sakamoto Y, Shimada K, Sano T, Kosuge T, Takahashi Y, et al. Arterial reconstruction during pancreatoduodenectomy in patients with celiac axis stenosis - utility of Doppler ultrasonography. World J Surg. 2005;29(7):885-9.

16. Crossin JD, Muradali D, Wilson SR. US of liver transplants: normal and abnormal. Radiographics. 2003;23(5):1093-114.

17. Dodd GD 3rd, Memel DS, Zajko AB, Baron RL, Santaguida LA. Hepatic artery stenosis and thrombosis in transplant recipients: Doppler diagnosis with resistive index and systolic acceleration time. Radiology. 1994;192(3):657-61.
Submit your next manuscript to BioMed Central and we will help you at every step:

- We accept pre-submission inquiries

- Our selector tool helps you to find the most relevant journal

- We provide round the clock customer support

- Convenient online submission

- Thorough peer review

- Inclusion in PubMed and all major indexing services

- Maximum visibility for your research

Submit your manuscript at www.biomedcentral.com/submit
Biomed Central 